ต

columns patients with psychotic illnesses developed stuttering while being treated with clozapine. The Government's Medicines and Healthcare Products Regulatory Agency (MHRA) is responsible for ensuring that medicines are acceptably safe. It takes action in relation to safety concerns and changes in the balances of risks and benefits. There is no mention by Lyall et al of informing the MHRA about the stuttering side-effect. I would like to urge readers of Psychiatric Bulletin to report to the MHRA any side-effects, suspected or otherwise, caused by a medicine through the Yellow Card scheme. This scheme plays an essential role in protecting public health by helping the MHRA to monitor the safety of medicines on the market. Psychiatrists and other healthcare professionals can complete a form online at www. yellowcard.gov.uk, or on a Yellow Card available in the British National Formulary, or directly from the MHRA (by telephoning 0800731 6789). I would also urge readers to encourage patients to report any side-effects. With these reports, we can actively look for signs of potential safety issues requiring further investigation

Reporting of adverse drug reactions is the professional duty of all healthcare professionals. The continued success of the Yellow Card Scheme depends on the continued support of health professionals and patients in completing Yellow Cards. We encourage Yellow Card reports from patients, but it is also vitally important that we continue to receive reports from psychiatrists and other health professionals.

June Raine Director of Vigilance and Risk Management of Medicines, Medicines and Healthcare Products RegulatoryAgency (MHRA), London SW8 5NQ, email: press.office@mhra.gsi.gov.uk doi: 10.1192/pb.31.5.191c

\section{Work-related stress in psychiatry}

I read with admiration Dr Harrison's report on work-related stress (Psychiatric Bulletin, October 2006, 30, 385-387) but I felt a sense of disappointment that we as psychiatrists and the wider medical profession continue to deny our health needs and general fallibility, and that our employers exhibit similar impotence.

Our training focuses our energies on succeeding both academically and later clinically. We are a competitive breed, entering our working life with high personal expectations of our performance on a day-to-day basis. The effect of daily consultation with morbidity and mortality on ourselves has to be addressed somehow. Denial becomes a handy defence mechanism.

As a profession we are more likely to develop alcohol misuse and dependence problems, as well as having a higher suicide rate. Yet how often do the precursors to these go unchecked or unnoticed. Taking time off sick is often accompanied by guilt and a sense of failure. We seem to believe that it shouldn't happen to us.

Currently, our junior doctors are in a heightened state of performance anxiety as Modernising Medical Careers goes live. The usual anxieties related to finding a job are magnified considerably by the number of jobs being applied for. How are we and our employers protecting this vital part of the work force from the inevitable stressrelated symptoms that are likely to ensue? When will we start to be honest with ourselves about our susceptibility to illness and look to prevent and manage it? When will our employers?

Amy M. Macaskill Specialist Registrar in Genera Adult Psychiatry, Royal Cornhill Hospital, 26 Cornhill Road, Aberdeen AB25 2ZH, email: amy.macaskill@ nhs.net

doi: 10.1192/pb.31.5.192

\section{How safe are interview rooms?}

Campbell \& Fung (Psychiatric Bulletin, January 2007, 31, 10-13) highlighted some important deficiencies in safety of patient interview rooms. We conducted a similar audit of 119 interview rooms in southern Hampshire in 2004, which was a repeat of an earlier study by local trainees in 2000 . We were therefore able to look at whether interview room safety had improved and whether newly commissioned facilities had been provided in accordance with the Department of Health's advice regarding the safety of interview rooms (Department of Health, 2004).

Our results were largely similar to Campbell \& Fung's but in southern Hampshire $75 \%$ of in-patient rooms were not isolated (v. 23\%), 75\% had a functioning panic alarm system (v. 0 ) and $52 \%$ had doors that opened outwards (v. 6\%). Of particular concern was that rooms used in accident and emergency departments to assess acutely disturbed and unknown patients were isolated, had no viewing window, no panic button and were cluttered.

It was reassuring to note that those rooms which had been commissioned in the past 3-4 years demonstrated a higher level of adherence to the standards: $92 \%$ had an unimpeded exit, 100\% had a functioning alarm and $77 \%$ had an internal inspection window. However, 67\% remained isolated and $61 \%$ were cluttered.

DEPARTMENT OF HEALTH (2004) Mental Health Policy Implementation Guide - Developing Positive Practice to Support the Safe and Therapeutic Management of Aggression and Violence in Mental Health In-Patient Settings. Department of Health.
Natasha Rae Specialist Registrar in Forensic Psychiatry, Wessex Forensic Rotation, Ravenswood House Medium Secure Unit, Knowle, Fareham P017 2NP, email: natasha.rae@hantspt-sw.nhs.uk Abigail Hewitt Specialist Registrar in Adult Psychiatry, St Anne's Hospital, Poole

doi: 10.1192/pb.31.5.192a

\section{BMA guidance on problem gambling}

The British Medical Association (BMA) has recently published a document on problem gambling in the UK prior to the Gambling Act 2005 coming fully into force in September. The document focuses on various aspects of problem gambling and particularly emphasises the potential impact on young people (British Medical Association, 2007). Also emphasised is the need for the National Health Service to provide help for those with this problem. Two important areas that the document does not emphasise however, are the impact on the elderly and their carers.

The UK has an ageing population, with $16 \%$ of the population currently aged over 65 . This is forecast to increase, with the elderly making up $19 \%$ of the population by 2021 (projected data from Office of Health Economics, 2002). The elderly can be at risk of problem gambling and are more likely to fall prey to the psychosocial consequences. It is therefore surprising that the BMA document did not make specific mention of this particularly vulnerable group of people.

The government has emphasised the importance of caring for carers (Department of Health, 1999). Carers of those with problem gambling could also suffer psychosocial distress and they require recognition and support. In the UK, where more and more elderly couples have only each other for support, this is particularly important. Again it is surprising that the BMA did not mention this in its recommendations.

BRITISH MEDICAL ASSOCIATION (2007) Gambling Addiction and its Treatment within the NHS: A Guide for Healthcare Professionals. BMA.

DEPARTMENT OFHEALTH (1999) Caring about Carers: A National Strategy for Carers. Department of Health.

Ishaan Gosai Specialist Registrar, Assertive Outreach Service, Plymouth, email: ishaang@ hotmail.co.uk

doi: $10.1192 / p b .31 .5 .192 b$

\section{Modified 'mooting' should be part of psychiatric training}

Naeem et al (Psychiatric Bulletin, January 2007, 31, 29-32) describe the incorporation of simulated mental health review tribunal workshops in psychiatric 
training. It is my experience that psychiatric trainees currently receive adequate preparation for successful participation in mental health review tribunals (MHRTs) via on the job experience under consultant supervision. However, the legal profession has long employed 'mooting' in their training. This involves a mock trial in which lawyers argue the legal principles of a fictitious case. The purpose is to improve analytical skills, the presentation of information and public speaking (http:// www.oup.co.uk/oxfordtextbooks/law/ mooting/more/). These principles can also be applied to giving oral evidence at a MHRT. Tribunals appear to be increasingly legalistic. In forensic psychiatry it is not uncommon for the patient to be represented by a senior barrister. Cross-examination of the doctor can be a lengthy, complex and stressful experience. The manner in which MHRTs are conducted in general psychiatry may also be affected in the future.

The proposed amendments to the Mental Health Act 1983 focus the doctor's role on issues that relate to the detention of patients and hence increase the importance of adequate formal training. Following the recent report of the Barrett homicide inquiry (NHS London, 2006) consultants may provide the only psychiatric input into MHRTs in the future, thus lessening training experience at work. Modified mooting or simulated MHRTs should be provided as part of psychiatric training, and should be introduced into both basic and higher training Liaison with lawyers would be important in designing these exercises. This might also produce more mutual understanding of professional roles.

NHS LONDON (2006) Report of the Independent Inquiry in the Care and Treatment ofJohn Barrett. NHS London.

Mark H. Taylor Specialist Registrar in Forensic Psychiatry, Fleming House, Rampton Hospital, Nottinghamshire DN22 OPD, email: Mark.Taylor2@ nottshc.nhs.uk

doi: $10.1192 / p b .31 .5 .192 c$

\section{Incentives for medication adherence}

As members of an assertive outreach team covering a socially deprived area of south-west Dublin, we read with interest the paper on money for medication by Claassen et al (Psychiatric Bulletin, January 2007, 31, 4-7) and congratulate the authors for applying contingency management measures, which are useful in other areas of medicine, in such an innovative, pragmatic way. Our team has not used financial incentives but has used other incentives to improve adherence to depot antipsychotics in a number of patients with severe illness and a high rate of hospitalisation. The incentives were negotiated with the patient and involved judicious and appropriate prescription of low doses of medications requested by the patient (such as lowdose hypnotics).

We discerned that the key ethical issues were undue influence and imbalance of power. We accept Claassen et al's differentiation of offer and threat, although we should point out that when a patient is taking medication for payment an implicit threat exists in that failure to continue results in a loss for the patient. However, against a background of several hospitalisations associated with serious reduction in quality of life because of nonadherence to effective medications, it does appear reasonable and ethical to regard the benefits to the patient of adherence as overcoming such negative factors as imperfect consent. We would counsel that such arrangements be subject to external review and monitoring.

*Guy Molyneux Specialist Registrar in Psychiatry Psychiatric Unit, Adelaide Meath National Children's Hospital, Dublin 24, email: guymoly@gmail.com, Patrick Devitt Consultant Psychiatrist, Adelaide Meath National Children's Hospital, Dublin

doi: 10.1192/pb.31.5.193

Claassen et al bring 'token economies' into the 21st century through offering financial incentives for single depot injections. The prejudices of the majority of team managers were not explored in detail. Of more interest is the process by which their 'operational policy' was developed and its progress through the local research ethics committee. How were the ethical considerations discussed by the authors addressed by the committee?

Two particular concerns arise from this study: the possible unwanted outcomes of payment and the process of discontinuation. The amount of payments 'depended on the frequency of depot injections'. It is not clear whether more or less frequent injections qualified for higher sums. Differential rewarding might have the unwanted effect of encouraging patients to modify their presentations to maximise payments. There is little possibility of discontinuation while payments are given for adherence and concordance is ignored. Claassen et al comment that adherence achieved through use of financial incentives may lead to greater insight and concordance. In a financially moribund National Health Service, it is likely that the small cost of paying patients to adhere to a treatment plan will obviate the need to provide more costly psychosocial interventions. This would be counterproductive and damaging to the clinical relationship.
Payments tied to a care plan that included psychosocial work with the aim of the patient moving into personally rewarding and possibly paid occupation would avoid problems associated with immediate payment based on adherence to medication. They could also provide an impetus to establishing concordance within a longer-term understanding of the recovery process.

Solmaz Sadaghiani Senior House Officer in Psychiatry, *Chris Fear Consultant Psychiatrist, Gloucestershire Partnership NHS Trust, Wotton Lawn Hospital, Horton Road, Gloucester GL1 3WL, email: chris.fear@glospart.nhs.uk

doi: 10.1192/pb.31.5.193a

Claassen et al state that financial incentives to increase adherence to depot medication should be considered further and welcome a debate upon the ethical implications of this management strategy. They identify autonomy, as defined by Beauchamp's four-principles approach, as a specific area of concern (Beauchamp, 2003). The key issue here therefore is whether the payment is coercive or not. If it is coercive, the individual's ability to act autonomously has been reduced. Claassen et al use Wertheimer's definition that 'threats coerce but offers do not', with a threat being an action that makes the individual worse off than a baseline whereas an offer does not (Wertheimer, 1993). They argue that the offer is not coercive and is therefore ethically neutral. It can be argued, however, that routine payment for receiving a depot will become the new baseline, rendering the removal of payment a threat. Removal of payment therefore becomes coercive, reducing autonomy.

The loss of money is minimised by Claassen et al because they consider its small value to mean that its removal is seen as non-threatening. However, this contradicts the hypothesis that it is the money that encourages the individual to accept the depot. Inevitably the use of payment will be coercive for some groups, reducing their autonomy. At the same time payment promotes access to essential treatment, in keeping with Beauchamp's principle of justice. A tension clearly exists between the principles of autonomy and justice; this is brought into sharper focus for disenfranchised, economically deprived groups.

BEAUCHAMP,T. L. (2003) Methods and principles in biomedical ethics. Journal of Medical Ethics, 29 $269-274$.

WERTHEIMER, A. (1993) A philosophical examination of coercion for mental health issues. Behavioural Sciences and the Law, 11, 239-258.

Giles Berrisford Specialist Registrar in Genera Adult Psychiatry, Queen Elizabeth Psychiatric Hospital, Edgbaston, Birmingham B15 2QX, email: giles.berrisford@bsmht.nhs.uk

doi: 10.1192/pb.31.5.193b 